\title{
When do wireless network signals appear Poisson?
}

\author{
H. PAUL KEELER ${ }^{1}$, NATHAN ROSS ${ }^{2, *}$ and AIHUA XIA ${ }^{2, * *}$ \\ ${ }^{1}$ Weierstrass Institute, Berlin 10117, Germany.E-mail: keeler@wias-berlin.de \\ ${ }^{2}$ School of Mathematics and Statistics, University of Melbourne, Parkville, VIC 3010, Australia. \\ E-mail: *nathan.ross@unimelb.edu.au; **aihuaxia@unimelb.edu.au
}

We consider the point process of signal strengths from transmitters in a wireless network observed from a fixed position under models with general signal path loss and random propagation effects. We show via coupling arguments that under general conditions this point process of signal strengths can be wellapproximated by an inhomogeneous Poisson or a Cox point processes on the positive real line. We also provide some bounds on the total variation distance between the laws of these point processes and both Poisson and Cox point processes. Under appropriate conditions, these results support the use of a spatial Poisson point process for the underlying positioning of transmitters in models of wireless networks, even if in reality the positioning does not appear Poisson. We apply the results to a number of models with popular choices for positioning of transmitters, path loss functions, and distributions of propagation effects.

Keywords: propagation loss; rate of convergence; signal strengths; total variation distance; weak convergence

\section{Introduction}

In this article, we study signal strengths in stochastic models of wireless networks such as ad hoc, sensor, and mobile or cellular phone networks. The building blocks of the models considered are point processes (from now on, we simply refer to them as processes) and stochastic geometry; for background, see $[1,2,15,16]$. Details of models can differ depending on the type of network, but the standard framework assumed throughout the article is that an observer is placed at the origin of $\mathbb{R}^{d}$ and transmitters are located at positions $\xi=\left\{x_{i}: i \in \mathbb{N}:=\{1,2, \ldots\}\right\} \subseteq \mathbb{R}^{d} \backslash\{0\}$ (equivalently we write $\xi=\sum_{i \in \mathbb{N}} \delta_{x_{i}}$, where $\delta_{x}$ is the Dirac measure at $x$ ) either deterministically or according to a random process. In the absence of propagation effects, the signal received by the observer from a transmitter located at $x \in \mathbb{R}^{d} \backslash\{0\}$ has strength given by a deterministic path loss function $\ell(x)$, typically taken to be a function of $|x|$, the distance to the origin; a standard assumption is $\ell(x)=C|x|^{-\beta}$ for some $\beta>0, C>0$ (though note that some papers refer to the reciprocal of $\ell$ as the path loss function to indicate that the path loss increases over distance). The random propagation effects are assumed to influence the strength of the signal via, for example, multipath fading (due to signals taking multiple paths and colliding with each other) and shadow fading or shadowing (due to signals colliding with large obstacles such as buildings). Although such effects may occur on different scales, we use the general term "fading" to refer to all types of random propagation effects, which are incorporated into the model via a sequence of i.i.d. positive random variables $S, S_{1}, S_{2}, \ldots$, where the signal power or strength from transmitter $x_{i}$ 
is given by

$$
P_{i}=\ell\left(x_{i}\right) S_{i}=: \frac{S_{i}}{g\left(x_{i}\right)} ;
$$

here and below $g(x):=1 / \ell(x)$. Understanding the distribution of the process

$$
\Pi:=\left\{P_{i}\right\}_{i \in \mathbb{N}}
$$

on $\mathbb{R}_{+}^{\circ}:=(0, \infty)$ and various functions of it (for example, the signal-to-interference ratio $\left\{P_{i} /\left(\sum_{j} P_{j}-P_{i}\right)\right\}_{i \in \mathbb{N}}$, or the largest signal strength $\left.\max _{i}\left\{P_{i}\right\}\right)$ under different assumptions on $S$, $g$ and $\xi$ is a major goal of wireless network modeling.

A common assumption is that the transmitter positions $\xi$ are given by a homogeneous Poisson process. In this case, easy theory implies that $\Pi$ is also a (typically inhomogeneous) Poisson process (easy theory means the Poisson marking and mapping theorems: the pairs $\left\{\left(x_{i}, S_{i}\right)\right\}_{x_{i} \in \xi}$ form a Poisson process [20], page 55, and the points of $\Pi$ are a measurable function of these points). The only potential difficulty with this framework is with computing the mean measure of $\Pi$ for explicit choices of $S$ and $g$. The assumption that the transmitters follow a Poisson process is usually justified by thinking of the observer as an "average" observer with fixed transmitters, though empirical studies are less clear on the matter [21]. Our main purpose is to investigate the behavior of $\Pi$ when the Poisson process transmitter assumption is relaxed and in particular to answer the titular question of the present article.

A first step is taken in [6,7] where it is shown that if $S=S(\sigma)=\exp \left\{\sigma B-\sigma^{2} / \beta\right\}$ for $B$ a standard normal random variable and $\beta>2, g(x)=(K|x|)^{\beta}$ for a constant $K>0$, and $\xi$ is such that $|\xi|(r)$, the number of points within distance $r$ of the origin, satisfies $|\xi|(r) /\left(\pi r^{2}\right) \rightarrow \lambda>$ 0 (a.s. if $\xi$ is random), then as $\sigma \rightarrow \infty$, $\Pi$ converges to a Poisson process. One of our main theorems is to greatly generalize this result, providing simple criteria for Poisson convergence.

A critical point before stating the theorem: to match empirical observation, the signal power process $\Pi$ should have a large number of very weak signals, which means any approximating Poisson process should have a mean measure with a (near) singularity at zero. To focus on the non-singular part, we instead study the process on $\mathbb{R}_{+}^{\circ}$ of the inverses of the signal power values,

$$
N=\left\{P_{i}^{-1}\right\}_{i \in \mathbb{N}}=\left\{\frac{g\left(x_{i}\right)}{S_{i}}\right\}_{i \in \mathbb{N}},
$$

though results about $N$ typically can be translated to results for $\Pi$ by inverting the points and using the Poisson mapping theorem; see, for example, Remark 2.3 below. The process $N$ on $\mathbb{R}_{+}^{\circ}$ is referred to as propagation (loss) process [8] (or path loss with fading process [14]) generated by $S, g$ and $\xi$. In the following theorem, we consider weak convergence of propagation processes in terms of the vague topology on the set of locally finite point measures on $\mathbb{R}_{+}^{\circ}$ [18], page 32 , denoted by $\stackrel{d}{\longrightarrow}$. We denote convergence in probability by $\stackrel{\mathbb{P}}{\longrightarrow}$.

Theorem 1.1. Let $\xi \subset \mathbb{R}^{d} \backslash\{0\}$ be a locally finite set of points such that there is a nondecreasing function $D$ satisfying (almost surely if $\xi$ is random)

$$
\lim _{r \rightarrow \infty} \frac{|\xi|(r)}{D(r)}=1 \quad \text { and } \quad \lim _{r \rightarrow 0} D(r)=0,
$$


and let $g: \mathbb{R}^{d} \rightarrow \mathbb{R}_{+}:=[0, \infty)$ such that $g$ is positive on $\mathbb{R}^{d} \backslash\{0\}$ and $g(x)=h(|x|)$ for $h$ a left continuous and nondecreasing function with inverse $h^{-1}(y):=\inf \{x: h(x)>y\}$. Let $(S(\sigma))_{\sigma \geq 0}$ be a family of positive random variables indexed by some non-negative parameter $\sigma, N^{(\sigma)}$ be the propagation process generated by $S(\sigma), g$ and $\xi$. If

$$
\text { (i) } S(\sigma) \stackrel{\mathbb{P}}{\longrightarrow} 0 \quad \text { and } \quad \text { (ii) } \mathbb{E} D\left(h^{-1}(S(\sigma) t)\right) \rightarrow L(t), \quad \forall t \in C(L),
$$

as $\sigma \rightarrow \infty$, where $C(L):=\left\{t \in \mathbb{R}_{+}^{\circ}: \lim _{s \rightarrow t} L(s)=L(t)\right\}$, then $N^{(\sigma)} \stackrel{d}{\longrightarrow} N^{(\infty)}$, where $N^{(\infty)}$ is a Poisson process on $\mathbb{R}_{+}^{\circ}$ with mean measure L, i.e., $\mathbb{E} N^{(\infty)}(d t)=d L(t)$.

It is straightforward to check that the function $L$ is non-decreasing, and in particular the set of discontinuities $\mathbb{R}_{+}^{\circ} \backslash C(L)$ is at most countable.

Remark 1.2. The result of [6], Theorem 3, already mentioned is an easy consequence of Theorem 1.1. Their assumptions are $d=2, S(\sigma)=\exp \left\{\sigma B-\sigma^{2} / \beta\right\}$ for $B$ a standard normal random variable and $\beta>2, g(x)=(K|x|)^{\beta}$ for a constant $K>0$, and $\xi$ is such that $|\xi|(r) /\left(\pi r^{2}\right) \rightarrow \lambda>0$. Thus we set $D(r)=\lambda \pi r^{2}$ and then noting that $\mathbb{E} S(\sigma)^{2 / \beta}=1$, Theorem 1.1 implies $N^{(\sigma)}$ converges weakly to a Poisson process with mean measure (here and below we abuse terminology for the sake of brevity and refer to a right-continuous non-decreasing function as a "mean measure" rather than writing "mean measure induced by")

$$
L(t)=\frac{\lambda \pi t^{2 / \beta}}{K^{2}} .
$$

Notice that with the same choices of $g$ and $\xi$, any $S(\sigma)$ such that $S(\sigma) \stackrel{\mathbb{P}}{\longrightarrow} 0$ and $\mathbb{E} S(\sigma)^{2 / \beta} \rightarrow 1$ has the same Poisson process limit as that of the sequence of lognormal random variables.

In particular, the same result holds for composite fading models [26] of product type having $S(\sigma)=S_{L}(\sigma) S_{F}$ with $S_{L}(\sigma) \stackrel{\mathbb{P}}{\longrightarrow} 0$ and $\mathbb{E} S_{L}(\sigma)^{2 / \beta} \rightarrow 1$ (for example the lognormal random variable above) and the random variable $S_{F}$ is independent of $S_{L}(\sigma)$ with $\mathbb{E} S_{F}^{2 / \beta}=1$. If $S_{F}$ is an exponential random variable with rate $\Gamma(1+2 / \beta)^{\beta / 2}$, and $S_{L}(\sigma)$ is the lognormal random variable above, then $S(\sigma)$ is known as a Suzuki model [26]. Actually, all that is required for convergence is $\mathbb{E} S_{F}^{2 / \beta}<\infty$ in which case $N^{(\sigma)}$ converges to a Poisson process with mean measure $L(t)=\lambda \mathbb{E} S_{F}^{2 / \beta} \pi t^{2 / \beta} / K^{2}$, cf., [7], Corollary 12 .

Moreover, by applying the Poisson mapping theorem, it's easy to see that the limiting Poisson process can also be realized by applying the function $g$ to the points of a homogenous Poisson process on $\mathbb{R}^{2}$ with intensity $\lambda$. Thus for modeling purposes, in many situations where Theorem 1.1 roughly applies (that is, the fading variables are small with sufficiently large probability) then one may assume that the underlying transmitter configuration is generated by a Poisson process.

Remark 1.3. Intuitively, because $S(\sigma)$ tends to zero, most points of $\xi$ are being sent out to infinity in $N^{(\sigma)}$. Large values of $S(\sigma)$ transform the far away points of $\xi$ closer to the origin, so a non-degenerate limit can only occur when, as $\sigma \rightarrow \infty, S(\sigma)$ becomes large on a set with 
probability inversely proportional to the number of points of $\xi$ at the appropriate distance. In particular, it's necessary that $\lim _{r \rightarrow \infty} D(r)=\infty$ to have a non-degenerate limit process (that is, with $L(t)$ not identically zero).

Remark 1.4. In essence, the Poisson limit is due to the thinning of the points in $\xi$. However, as the retained points are redistributed, the thinning scheme in this paper is very different from the classical thinning schemes in the literature, for example, [11,17,28,29].

Remark 1.5. The condition (1.3) on $\xi$ is satisfied for transmitters placed on a regular lattice excluding the origin or that are a realization of a stationary, ergodic process with no points at the origin; in the former case we would take $D(r)$ proportional to the volume of a ball of radius $r$ in $d$ dimensions.

Theorem 1.1 is one of a number of approximation and convergence results we establish in this paper that apply to the inverse signal strength process $N$; we state these in detail in Section 2. In Section 3, we provide an overview of some standard wireless network models and apply our results to these. Section 4 contains proofs and we conclude the paper with some discussion in Section 5.

\section{Poisson process approximating signal powers}

We state our formal setup.

Setup 2.1 (Main setup). Let $\xi \subset \mathbb{R}^{d} \backslash\{0\}$ be a locally finite collection of points in $\mathbb{R}^{d}$ and $g$ be a measurable mapping from $\left(\mathbb{R}^{d}, \mathcal{B}\left(\mathbb{R}^{d}\right)\right)$ to $\left(\mathbb{R}_{+}, \mathcal{B}\left(\mathbb{R}_{+}\right)\right)$such that $\{g(x): x \in \xi\}$ is locally finite; here $\mathcal{B}(\cdot)$ denotes the Borel $\sigma$-algebra. Write $\xi=\left\{x_{i}: i \in \mathcal{I}^{\xi}\right\}$ where $\mathcal{I}^{\xi}$ is a finite or countable index set (without loss, taken to be $\{1, \ldots, n\}$ in the finite case and $\mathbb{N}$ in the infinite case). Define $|\xi|(r)$ as the number of points of $\xi$ within distance $r$ of the origin and let $\left\{S, S_{i}: i \in \mathcal{I}^{\xi}\right\}$ be a sequence of independent and identically distributed positive random variables. Set $Y_{i}=g\left(x_{i}\right) / S_{i}$ and let $N$ be the propagation process generated by the collection $\left\{Y_{i}\right\}_{i \in \mathcal{I} \xi}$, that is, $N=\sum_{i \in \mathcal{I}^{\xi}} \delta_{Y_{i}}$. Let $p^{(x)}(t)=\mathbb{P}(0<g(x) / S \leq t), x \in \mathbb{R}^{d}, p_{i}(t)=p^{\left(x_{i}\right)}(t), M(t):=M^{\xi}(t):=\sum_{i \in \mathcal{I}^{\xi}} p_{i}(t)$ and $Z$ be a Poisson process on $\mathbb{R}_{+}^{\circ}$ having mean measure $M(t)$. We assume $M(t)<\infty$ for all $t>0$ so that $Z$ is well-defined. For any Radon measure $\eta$ on $\mathbb{R}_{+}^{\circ}$ and $\tau>0$, we define $\eta(\tau):=\eta((0, \tau])$ and $\left.\eta\right|_{\tau}$ as the Radon measure restricted to the interval $(0, \tau]$.

Recall the total variation distance between two probability measures $v_{1}, v_{2}$ on the same measurable space $(\mathcal{D}, \mathcal{F}(\mathcal{D}))$ is defined as

$$
d_{\mathrm{TV}}\left(v_{1}, v_{2}\right)=\sup _{A \in \mathcal{B}(\mathcal{D})}\left|v_{1}(A)-v_{2}(A)\right| .
$$

Total variation distance is a common and strong metric that bounds the maximum difference in probabilities between two probability distributions. In our context, we take $\mathcal{D}=\mathcal{H}$, the space of 
all locally finite point measures on $\mathbb{R}_{+}^{\circ}$ or $\mathbb{R}^{d}$ equipped with the vague topology [18], page 169, and $\mathcal{F}(\mathcal{D})=\mathcal{B}(\mathcal{H})$, the Borel $\sigma$-algebra generated by the vague topology.

We now present a bound on the total variation distance between the distributions of processes $\left.N\right|_{\tau}$ and $\left.Z\right|_{\tau}$, where $1 / \tau$ can be interpreted as the smallest possible power value of interest for an observer in the network. Here and below we use $\mathcal{L}(X)$ to denote the distribution of a random element $X$.

Theorem 2.2. Assuming the main Setup 2.1, we have

$$
\frac{1 \wedge M(\tau)^{-1}}{32} \sum_{i \in \mathcal{I}^{\xi}} p_{i}(\tau)^{2} \leq d_{\mathrm{TV}}\left(\mathcal{L}\left(\left.Z\right|_{\tau}\right), \mathcal{L}\left(\left.N\right|_{\tau}\right)\right) \leq \sum_{i \in \mathcal{I}^{\xi}} p_{i}(\tau)^{2} \leq M(\tau) \sup _{i \in \mathcal{I}^{\xi}} p_{i}(\tau) .
$$

Remark 2.3. Since the total variation distance is preserved under one to one mappings, Theorem 2.2 also implies that for $Z^{\prime}$ the Poisson process with mean measure $M^{\prime}[\tau, \infty)=M(1 / \tau)$, and denoting the restriction of $Z^{\prime}$ to $[\tau, \infty)$ by $\left.Z^{\prime}\right|^{\tau}$,

$$
d_{\mathrm{TV}}\left(\mathcal{L}\left(\left.Z^{\prime}\right|^{\tau}\right), \mathcal{L}\left(\left.\Pi\right|^{\tau}\right)\right) \leq \sum_{i \in \mathcal{I}^{\xi}} p_{i}(1 / \tau)^{2} \leq M(1 / \tau) \sup _{i \in \mathcal{I}^{\xi}} p_{i}(1 / \tau),
$$

where $\Pi$ is the process of signal powers defined in the introduction.

Remark 2.4. Theorem 2.2 shows that the main criterion for Poisson process convergence in the wireless network setting is that, in the notation of the theorem, $\sup _{i \in \mathcal{I}^{\xi}} p_{i}(\tau)$ is small. The other criterion is the convergence of the mean measure, though the theorem still technically applies even if $M(t)$ is infinite or zero.

Theorem 2.2 is an approximation theorem, but we can use it to prove weak convergence results; note that such convergence implies convergence of natural statistics of the process, e.g., point counts for finite collections of relatively compact Borel sets having no points on the boundaries almost surely in the limit and random variables that are integrals against the point measure of continuous and compactly supported functions $f: \mathbb{R}_{+}^{\circ} \rightarrow \mathbb{R}_{+}$[18], Theorem 4.2. We have the following corollary of Theorem 2.2.

Corollary 2.5. For each $n$, let $\xi_{n}=\left\{x_{n i}\right\}_{i \in \mathcal{I}_{n}}, \mathcal{I}_{n}:=\mathcal{I}^{\xi_{n}}, S_{n i}$ and $Y_{n i}$ satisfy the main Setup 2.1, and define $N^{(n)}$ as the process generated by the $\left\{Y_{n i}\right\}_{i \in \mathcal{I}_{n}}$. If for all $t \in C(L)$,

$$
\lim _{n \rightarrow \infty} \sup _{i \in \mathcal{I}_{n}} \mathbb{P}\left(0<Y_{n i} \leq t\right)=0 \quad \text { and } \quad \lim _{n \rightarrow \infty} \mathbb{E} N^{(n)}(t)=L(t),
$$

then $N^{(n)} \stackrel{d}{\longrightarrow} Z^{L}$, where $Z^{L}$ is a Poisson process on $\mathbb{R}_{+}^{\circ}$ with mean measure $L$.

The caveat to Theorem 2.2 and Corollary 2.5 is that the mean measure of the processes may be difficult to compute in practice. The following proposition provides alternative formulas for the mean measure. Recall that for a non-decreasing function $h$ we define the inverse $h^{-1}(y)=$ $\inf \{x: h(x)>y\}$. 
Proposition 2.6. Recall the notation of the main Setup 2.1 and assume $g(x)=h(|x|)$ for some function $h$. Then

$$
M(t):=M^{\xi}(t):=\sum_{i \in \mathcal{I}^{\xi}} p_{i}(t)=\int_{0}^{\infty} \mathbb{P}\left(0<\frac{h(r)}{S} \leq t\right)|\xi|(d r) .
$$

If in addition, $h$ is positive on $\mathbb{R}_{+}^{\circ}$, left continuous and nondecreasing with inverse $h^{-1}$, then

$$
M(t)=\mathbb{E}\left[|\xi|\left(h^{-1}(S t)\right)\right]
$$

\subsection{Random transmitter positions}

If the transmitters are placed according to a random process $\Xi$ independent of the fading sequence, then Theorem 2.2 holds conditionally, but the mean measure of the approximating Poisson process may change for different realizations of $\Xi$ and so in general we approximate $N$ by a Cox process, which is a Poisson process with a random intensity measure. Note that even in this case, each user sees a Poisson process, but the intensity measures of users in different locations and at different times may be different.

We change the main Setup 2.1 by replacing $\xi$ with a process $\Xi$. According to [18], Lemma 2.3, we can write $\Xi=\sum_{i \in \mathcal{I}^{\Xi}} \delta_{X_{i}}$ with (possibly random) index set $\mathcal{I}^{\Xi}$. Again, we let $\left\{S, S_{i}: i \in \mathbb{N}\right\}$ be a sequence of positive i.i.d. random variables independent of $\Xi$ and we define $N:=N^{\Xi}$ as in the main Setup 2.1 but with $\Xi$ replacing $\xi$.

Before stating our Cox process approximation result, we cover the important case where $\Xi$ is approximately a Poisson process, in which case Poisson process approximation is valid.

Theorem 2.7. With the setup above, let $\Theta$ be a Poisson process on $\mathbb{R}^{d}$ independent of $\left\{S_{i}: i \in \mathbb{N}\right\}$ and define $Z=\sum_{\theta_{i} \in \Theta} \delta_{g\left(\theta_{i}\right) / S_{i}}$, as a process on $\mathbb{R}_{+}^{\circ}$. Then $Z$ is a Poisson process with mean measure

$$
M(t)=\mathbb{E} Z(t)=\mathbb{E} \int \mathbb{P}(0<g(\theta) / S \leq t) \Theta(d \theta)
$$

and

$$
d_{\mathrm{TV}}\left(\mathcal{L}\left(\left.Z\right|_{\tau}\right), \mathcal{L}\left(\left.N\right|_{\tau}\right)\right) \leq d_{\mathrm{TV}}(\mathcal{L}(\Theta), \mathcal{L}(\Xi))
$$

Theorem 2.7 shows that if $\Xi$ is close to some Poisson process, then $N$ is close to a Poisson process. In general, random $\Xi$ give rise to Cox processes which are Poisson processes with random mean measures. More precisely, we say that $Z$ is a Cox process directed by the random measure $M$ if conditional on $M, Z$ is a Poisson process with mean measure $M$. We have the following result.

Theorem 2.8. Recall the main Setup 2.1, but with $\xi$ replaced by a locally finite process $\Xi$ independent of $\left\{S_{i}\right\}_{i \in \mathbb{N}}$. Define

$$
M^{\Xi}(t)=\int_{\mathbb{R}^{d}} p^{(x)}(t) \Xi(d x)
$$


Let $Z$ be the Cox process directed by the measure $M^{\Xi}$, that is, conditional on $\Xi, Z$ is a Poisson process with mean measure $M^{\Xi}$. Then

$$
d_{\mathrm{TV}}\left(\mathcal{L}\left(\left.Z\right|_{\tau}\right), \mathcal{L}\left(\left.N\right|_{\tau}\right)\right) \leq \mathbb{E} \int_{\mathbb{R}^{d}} p^{(x)}(\tau)^{2} \Xi(d x) .
$$

The following conditional and unconditional analog of Proposition 2.6 holds for random $\xi$; we omit the proof because it's straightforward from Proposition 2.6.

Proposition 2.9. Recall the notation of the main Setup 2.1 with $\xi$ replaced by a locally finite process $\Xi$ independent of $S_{1}, S_{2}, \ldots$ and assume $g(x)=h(|x|)$ for some function $h$. Then

$$
M^{\Xi}(t):=\int_{\mathbb{R}^{d}} p^{(x)}(t) \Xi(d x)=\int_{0}^{\infty} \mathbb{P}\left(0<\frac{h(r)}{S} \leq t\right)|\Xi|(d r)
$$

and

$$
M(t):=\int_{\mathbb{R}^{d}} p^{(x)}(t) \Lambda(d x)=\mathbb{E} \int_{0}^{\infty} \mathbb{P}\left(0<\frac{h(r)}{S} \leq t\right)|\Xi|(d r),
$$

where $\Lambda$ is the mean measure of $\Xi$. If in addition, $h$ is positive on $\mathbb{R}_{+}^{\circ}$, left continuous and nondecreasing with inverse $h^{-1}$, then

$$
M^{\Xi}(t)=\mathbb{E}\left[|\Xi|\left(h^{-1}(S t)\right) \mid \Xi\right]
$$

and

$$
M(t)=\mathbb{E}\left[|\Xi|\left(h^{-1}(S t)\right)\right]=\mathbb{E}\left[|\Lambda|\left(h^{-1}(S t)\right)\right],
$$

where $|\Lambda|(r):=\mathbb{E}|\Xi|(r)$.

\subsection{Poisson versus Cox}

When the transmitters are randomly placed according to a process $\Xi$, it's possible for the propagation process $N$ to be close to a Poisson process (Theorem 2.7) or Cox process with nondeterministic mean measure (Theorem 2.8). There are realistic situations where we expect the propagation process to be close to a Cox process, for example, if the fading distribution $S$ is a mixture of distributions (see Section 3.2 for an example) or the observer initially connects in a random way to one of a number of different networks that have different transmitter coverage densities or fading distributions (see, the example (iii) below, for a toy model). However, it's of interest to understand when a Poisson, rather than Cox, process is appropriate. Theorem 2.8 suggests that if $M^{\Xi}(t)$ is close to deterministic, then $N$ may be close to Poisson. The next result is a convergence version of this statement with easily checkable conditions.

Theorem 2.10. Assume that $\Xi$ is a process on $\mathbb{R}^{d}$ with a locally finite mean measure $\Lambda$ such that $\lim _{r \downarrow 0}|\Lambda|(r)=0$ and as $r \rightarrow \infty$,

$$
|\Lambda|(r) \rightarrow \infty, \quad \operatorname{Var}(|\Xi|(r)) /(|\Lambda|(r))^{2} \rightarrow 0 .
$$


Let $(S(\sigma))_{\sigma \geq 0}$ be a family of positive random variables, $N^{(\sigma)}$ be the propagation process generated by $S(\sigma), g$ and $\Xi$. Assume $g(x)=h(|x|)$, where $h$ is left continuous, nondecreasing and positive on $\mathbb{R}_{+}^{\circ}$. If
(i) $S(\sigma) \stackrel{\mathbb{P}}{\longrightarrow} 0$ and
(ii) $\int_{\mathbb{R}^{d}} \mathbb{P}\left(0<\frac{g(x)}{S(\sigma)} \leq t\right) \Lambda(d x) \rightarrow L(t)$
for all $t \in C(L)$

as $\sigma \rightarrow \infty$, then $N^{(\sigma)}$ converges weakly to a Poisson process $Z^{L}$ with mean measure $L$.

We provide a few easy examples to illustrate the result; Example (iii) shows that if (2.6) is not satisfied then in general we can't say the limit is Poisson.

\section{Example 2.11.}

(i) If $\Xi=\xi$ is non-random, $\operatorname{Var}(|\Xi|(r))=0$ and Theorem 2.10 reiterates Theorem 1.1 that the limit process is Poisson.

(ii) If $\Xi$ is a Poisson process with intensity $\lambda$, then $\operatorname{Var}(|\Xi|(r)) /(\mathbb{E}|\Xi|(r))^{2}=\left(\lambda \pi r^{2}\right)^{-1}$ which tends to zero as $r \rightarrow \infty$, so the limit process is Poisson.

(iii) If $\Xi$ is a Cox process having intensity $\lambda_{i}>0$ with probability $1 / 2$ for $i=1,2$ and $\lambda_{1} \neq \lambda_{2}$, then it is clear from Theorem 2.7 that $N$ is a Cox process directed by a random mean measure that takes two measures with equal probability. Using the generic formula $\operatorname{Var}(X)=\mathbb{E} \operatorname{Var}[X \mid \mathcal{A}]+$ $\operatorname{Var}(\mathbb{E}[X \mid \mathcal{A}])$, valid for any random variable $X$ and sigma-algebra $\mathcal{A}$, we can derive that

$$
\mathbb{E}|\Xi|(r)=\frac{\lambda_{1}+\lambda_{2}}{2} \pi r^{2}, \quad \operatorname{Var}(|\Xi|(r))=\frac{\lambda_{1}+\lambda_{2}}{2} \pi r^{2}+\frac{\left(\lambda_{1}-\lambda_{2}\right)^{2}}{4} \pi^{2} r^{4},
$$

and hence $\lim _{r \downarrow 0}|\Lambda|(r)=0, \lim _{r \rightarrow \infty}|\Lambda|(r)=\infty$ but

$$
\lim _{r \rightarrow \infty} \frac{\operatorname{Var}(|\Xi|(r))}{(\mathbb{E}|\Xi|(r))^{2}}=\frac{\left(\lambda_{1}-\lambda_{2}\right)^{2}}{\left(\lambda_{1}+\lambda_{2}\right)^{2}} \neq 0
$$

\section{Applications}

We assume throughout this section that $g$ is a function on $\mathbb{R}^{d}$ such that $g$ is positive on $\mathbb{R}^{d} \backslash\{0\}$ and $g(x)=h(|x|)$ for $h$ a left continuous and nondecreasing function with inverse $h^{-1}$. We apply Theorem 1.1 to fading tending to zero (Section 3.1), Theorem 2.8 to a composite model (Section 3.2), Theorem 2.7 to transmitters placed according to a Poisson process (Section 3.3), and Theorem 2.10 to transmitters placed according to an $\alpha$-Ginibre process (Section 3.4 where $\alpha$-Ginibre processes are defined). 


\subsection{Fading tending to zero}

As already discussed in Remark 1.2, Theorem 1.1 can be applied to many standard models. We first demand that $\xi$ is such that there is a nondecreasing function $D$ satisfying

$$
\lim _{r \rightarrow \infty} \frac{|\xi|(r)}{D(r)}=1 \quad \text { and } \quad \lim _{r \rightarrow 0} D(r)=0 .
$$

This condition is satisfied for lattices on $\mathbb{R}^{d}$, stationary and ergodic processes. In particular, specializing to the case $d=2$, if transmitters are at the vertices of a

- triangular lattice, edge lengths $s$, excluding the origin, then $D(r)=2 \pi r^{2} /\left(\sqrt{3} s^{2}\right)$;

- hexagonal lattice, edge lengths $s$, excluding the origin, then $D(r)=4 \pi r^{2} /\left(3 \sqrt{3} s^{2}\right)$;

- square lattice, edge lengths $s$, excluding the origin, then $D(r)=\pi r^{2} / s^{2}$.

From this point, for a given $h$, we only need to have $S(\sigma) \stackrel{\mathbb{P}}{\longrightarrow} 0$ and $\mathbb{E} D\left(h^{-1}(S(\sigma) t)\right) \rightarrow L(t)$ for $t \in C(L)$. Computing this expectation is straightforward under nice distributions of $S(\sigma)$; even for composite models, for example, when $S(\sigma)$ is a product of independent variables. Finally, it's worth repeating that if $L$ equals zero or infinity, the theorem is still true (with the obvious interpretation of a Poisson with mean zero or infinity) though not of practical interest.

\subsection{Dependent composite fading}

In Remark 1.2, we showed that if the transmitters are placed on $\mathbb{R}^{2}, g(x)=(K|x|)^{\beta}$ for $\beta>$ $2, K>0, \xi$ is such that $|\xi|(r) / r^{2} \rightarrow \lambda \pi$, and the composite fading distribution is

$$
S(\sigma)=\exp \left\{\sigma B-\sigma^{2} / \beta\right\} S_{F},
$$

where $B$ is a standard normal random variable independent of $S_{F}$, and $S_{F}$ is exponential with rate $\Gamma(1+2 / \beta)^{\beta / 2}$ (or any other random variable such that $\mathbb{E} S_{F}^{2 / \beta}=1$ ), then $N^{(\sigma)}$ converges to Poisson process with mean measure

$$
M(t)=\frac{\lambda \pi t^{2 / \beta}}{K^{2}}
$$

(The rate of the exponential is only a scaling factor and convergence only demands that $\mathbb{E} S_{F}^{2 / \beta}$ be finite, though this changes the mean measure.) Now assume instead that each $S_{i}(\sigma), i=1,2, \ldots$ is distributed as $S(\sigma)$, but rather than being i.i.d. they share a common $S_{F}$ variable which is not necessarily exponential. That is, define

$$
S_{i}=\exp \left\{\sigma B_{i}-\sigma^{2} / \beta\right\} S_{F},
$$


where $B_{1}, B_{2}, \ldots$ are i.i.d. standard normal random variables. Then conditional on $S_{F}, N^{(\sigma)}$ converges to a Poisson process with mean measure

$$
M^{S_{F}}(t):=\frac{S_{F}^{2 / \beta} \lambda \pi t^{2 / \beta}}{K^{2}} .
$$

Thus $N^{(\sigma)}$ converges to a Cox process directed by $M^{S_{F}}$.

\subsection{Transmitters placed according to a Poisson process}

As mentioned in the introduction, a common assumption is that the locations of transmitters $\Xi$ follow a Poisson process. If $\Xi$ is a Poisson process then Theorem 2.7 shows that $N$ is a Poisson process. Moreover, the mean measure of $N$ is computed through (2.5). We compute the mean measure in some examples.

Example A. If $\Xi$ is a homogeneous Poisson process with intensity $\lambda, g(x)=(K|x|)^{\beta}$, for some $\beta>0, K>0$, then it's well known (see [9], Section I.A) that $N$ is a Poisson process with intensity measure depending on $S$ only through $\mathbb{E} S^{2 / \beta}$, which has been referred to as propagation invariance. So $h(r)=K^{\beta} r^{\beta}$ and the mean measure as given by (2.2) is

$$
M(t)=\lambda \pi \mathbb{E}\left[h^{-1}(S t)^{2}\right]=\lambda \pi t^{2 / \beta} \mathbb{E}\left[S^{2 / \beta}\right] / K^{2} .
$$

Example B. More generally, if $h(r)=r^{\beta} e^{\alpha r}$ (used in the empirical work [13]) and $W$ is the Lambert $W$-function, that is, $x=W(y)$ is the solution of $x e^{x}=y$, then $h(r)=x$ gives $r=h^{-1}(x):=\frac{\beta}{\alpha} W\left(\alpha x^{1 / \beta} / \beta\right)$, so Proposition 2.9 implies

$$
\begin{aligned}
M(t) & =\pi \lambda \mathbb{E}\left(\frac{\beta}{\alpha} W\left(\frac{\alpha}{\beta}(t S)^{1 / \beta}\right)\right)^{2} \\
& =\pi \lambda \mathbb{E}\left[(t S)^{2 / \beta} e^{-2 W\left(\frac{\alpha}{\beta}(t S)^{1 / \beta}\right)}\right],
\end{aligned}
$$

where the second equality uses the fact that $W(y)^{2}=y^{2} e^{-2 W(y)}$.

Example C. Generalizations of the power-law path loss function are so-called multi-slope models which have

$$
h(r)=\left(\sum_{i=1}^{k+1} \mathbf{1}_{r_{i-1} \leq r<r_{i}} b_{i} r^{-\beta_{i}}\right)^{-1}=\sum_{i=1}^{k+1} \mathbf{1}_{r_{i-1} \leq r<r_{i}} b_{i}^{-1} r^{\beta_{i}},
$$

where 1 is the indicator function, $0=r_{0}<r_{1}<\cdots<r_{k}<r_{k+1}=\infty, \beta_{i}>0$, and $b_{i}>0$ are chosen to make $h$ continuous; see [31] and references there. Since each interval $\left[r_{i-1}, r_{i}\right)$ is disjoint with all others, the inverse of the multi-slope model is simply

$$
h^{-1}(s)=\sum_{i=1}^{k+1} \mathbf{1}_{s_{i-1} \leq s<s_{i}} c_{i} s^{1 / \beta_{i}}
$$


where $s_{i}=b_{i}^{-1} r_{i}^{\beta_{i}}$ and $c_{i}=b_{i}^{1 / \beta_{i}}$. Theorem 2.7 says that the propagation process is Poisson and according to expression (2.5), the mean measure is

$$
M(t)=2 \pi \lambda \mathbb{E}\left(h^{-1}(t S)^{2}\right)=2 \pi \lambda \sum_{i=1}^{k} t^{2 / \beta_{i}} c_{i} \mathbb{E}\left[\mathbf{1}_{s_{i-1} \leq t S<s_{i}} S^{2 / \beta_{i}}\right] .
$$

Note that in contrast to the propagation invariance of the case of the power-law path loss function of Example B above, where all $S$ with the same $2 / \beta$ moment induce a common propagation process distribution, the form of the mean measure (3.1) suggests that no analogous simple invariance property holds for the multi-slope model.

\subsection{Transmitters placed as an $\alpha$-Ginibre process}

The assumption that transmitters are placed according to a Poisson process can be heuristically justified by considering an "average" observer with fixed transmitters and is a convenient assumption due to its tractability. There has been recent interest in modeling the transmitter locations according to other processes, especially those that exhibit repulsion or clustering among the points (some networks are designed to resemble lattices while others have clustering due to physical and technological considerations) [10]. One such process that exhibits repulsion is the $\alpha$-Ginibre process on the complex plane $\mathbb{C}$ which has been used to model mobile networks [23], [24]. The process is defined through the factorial moment measures: for a locally finite process $\Xi$ on a Polish space $\mathcal{S}$, the $n$th order factorial moment measure $v^{(n)}$ of $\Xi$ is defined by the relation [18], pages 109-110 (also see [1], Chapter 9), where $f$ ranges over all Borel measurable functions $h: \mathcal{S}^{n} \rightarrow[0, \infty)$. The special case $v^{(1)}$ is simply the mean measure of $\Xi$. To define the Ginibre process, for $x \in \mathbb{C}$, let $\bar{x}$ and $|x|$ be the complex conjugate and modulus of $x$.

Definition 3.1. We say the process $\Xi$ on the complex plane $\mathbb{C}$ is an $\alpha$-Ginibre process if its factorial moment measures are given by

$$
v^{(n)}\left(d x_{1}, \ldots, d x_{n}\right)=\rho^{(n)}\left(x_{1}, \ldots, x_{n}\right) d x_{1} \cdots d x_{n}, \quad n \geq 1,
$$

where $\rho^{(n)}\left(x_{1}, \ldots, x_{n}\right)$ is the determinant of the $n \times n$ matrix with $(i, j)$ th entry

$$
K_{\alpha, c}\left(x_{i}, x_{j}\right)=\frac{c}{\pi} e^{-\frac{c}{2 \alpha}\left(\left|x_{i}\right|^{2}+\left|x_{j}\right|^{2}\right)} e^{\frac{c}{\alpha} x_{i} \bar{x}_{j}}, \quad c>0 .
$$

In particular, direct computation gives

$$
\rho^{(1)}(x)=\frac{c}{\pi}>0 \quad \text { and } \quad \rho^{(2)}(x, y)=\frac{c^{2}}{\pi^{2}}\left(1-e^{-\frac{c}{\alpha}|x-y|^{2}}\right) .
$$

Theorem 3.2. Assume that $\Xi$ is an $\alpha$-Ginibre process on $\mathbb{C},(S(\sigma))_{\sigma \geq 0}$ is a family of positive random variables, $g$ is a function such that $g$ is positive on $\mathbb{R}^{d} \backslash\{0\}$ and $g(x)=h(|x|)$, with 
$h$ a left continuous and nondecreasing function with inverse $h^{-1}$, and $N^{(\sigma)}$ is the propagation process generated by $S(\sigma), g$ and $\Xi$. If
(i) $S(\sigma) \stackrel{\mathbb{P}}{\longrightarrow} 0$ and
(ii) $\lambda \pi \mathbb{E}\left[h^{-1}(S(\sigma) t)^{2}\right] \rightarrow L(t)$
for all $t \in C(L)$

as $\sigma \rightarrow \infty$, then $N^{(\sigma)}$ converges weakly to a Poisson process $Z^{L}$ with mean measure $L$.

Proof of Theorem 3.2. We show $\operatorname{Var}(|\Xi|(r)) /(\mathbb{E}|\Xi|(r))^{2} \rightarrow 0$ as $r \rightarrow \infty$ and then the result follows by Theorem 2.10. Now, let $C_{r}=\{x \in \mathbb{C}:|x| \leq r\}$, we find

$$
\begin{aligned}
\mathbb{E}\left[|\Xi|(r)^{2}\right] & =\mathbb{E} \int_{C_{r}} \int_{C_{r}} \Xi(d x)\left(\Xi-\delta_{x}\right)(d y)+\mathbb{E} \int_{C_{r}} \Xi(d x) \\
& =\int_{C_{r}} \int_{C_{r}} \frac{c^{2}}{\pi^{2}}\left(1-e^{-\frac{c}{\alpha}|x-y|^{2}}\right) d x d y+\int_{C_{r}} \frac{c}{\pi} d x \\
& \leq c^{2} r^{4}+c r^{2} .
\end{aligned}
$$

Since $\mathbb{E}|\Xi|(r)=c r^{2}$, we find that $\operatorname{Var}(|\Xi|(r)) \leq c r^{2}$ and $\operatorname{Var}(|\Xi|(r)) /(\mathbb{E}|\Xi|(r))^{2} \leq 1 /\left(c r^{2}\right) \rightarrow 0$ as $r \rightarrow \infty$.

For example, choosing $g(x)=(K|x|)^{\beta}$ for $\beta>2, K>0$, and

$$
S(\sigma)=\exp \left\{\sigma B-\sigma^{2} / \beta\right\} S_{F}
$$

where $B$ is standard normal random variable, independent of $S_{F}$ and which satisfies $\mathbb{E} S_{F}^{2 / \beta}=1$ (for example, $S_{F}=1$ or is exponential with rate $\Gamma(1+2 / \beta)^{\beta / 2}$ ) then $N^{(\sigma)}$ converges to Poisson process with mean measure

$$
M(t)=\frac{\lambda \pi t^{2 / \beta}}{K^{2}} .
$$

\section{Proofs}

Proof of Theorem 2.2. We first show the upper bounds. Let $Z_{i}$ be independent Poisson processes on $\mathbb{R}_{+}^{\circ}$ with mean measures $p_{i}(t)$ and $N_{i}$ be the process placing a single point at $Y_{i}$. Notice that

$$
\sum_{i \in \mathcal{I}^{\xi}} Z_{i} \stackrel{d}{=} Z, \quad \text { and } \quad \sum_{i \in \mathcal{I}^{\xi}} N_{i} \stackrel{d}{=} N
$$

where $\stackrel{d}{=}$ means they are equal in distribution, and by independence we can bound

$$
d_{\mathrm{TV}}\left(\mathcal{L}\left(\left.Z\right|_{\tau}\right), \mathcal{L}\left(\left.N\right|_{\tau}\right)\right) \leq \sum_{i \in \mathcal{I}^{\xi}} d_{\mathrm{TV}}\left(\mathcal{L}\left(\left.Z_{i}\right|_{\tau}\right), \mathcal{L}\left(\left.N_{i}\right|_{\tau}\right)\right)
$$


Straightforward considerations show that for both $\left.Z_{i}\right|_{\tau}$ and $\left.N_{i}\right|_{\tau}$, given there is a single point in the interval $(0, \tau]$, it is distributed with density

$$
\frac{p_{i}(d t)}{p_{i}(\tau)}, \quad 0<t \leq \tau .
$$

An alternative expression for the total variation distance, using Monge-Kantorovich duality [25], is

$$
d_{\mathrm{TV}}\left(v_{1}, v_{2}\right)=\inf _{\left(\xi_{1}, \xi_{2}\right)} \mathbb{P}\left(\xi_{1} \neq \xi_{2}\right),
$$

where the infimum is over all couplings of $v_{1}, v_{2}$. So from the previous consideration, if $Z_{i}(\tau)=$ $N_{i}(\tau)$ (as above, $Z_{i}(\tau)$ is the number of points of $Z_{i}$ in the interval $\left.(0, \tau]\right)$, then we can couple $\left.Z_{i}\right|_{\tau}$ and $\left.N_{i}\right|_{\tau}$ exactly. Thus, we easily find

$$
d_{\mathrm{TV}}\left(\mathcal{L}\left(\left.Z_{i}\right|_{\tau}\right), \mathcal{L}\left(\left.N_{i}\right|_{\tau}\right)\right)=d_{\mathrm{TV}}\left(\mathcal{L}\left(Z_{i}(\tau)\right), \mathcal{L}\left(N_{i}(\tau)\right)\right) \leq p_{i}(\tau)^{2}
$$

the last inequality follows by noting that $Z_{i}(\tau)$ is Poisson with mean $p_{i}(\tau)$ and $N_{i}(\tau)$ is a Bernoulli with success probability $p_{i}(\tau)$ and then using well-known bounds between Poisson random variables and Bernoullis [22]; see also [5], Formula (1.8). Combining (4.1) and (4.2) proves the first upper bound of the theorem and the second is simple.

For the lower bound, note that the number of points of $N$, respectively $Z$, falling in $(0, \tau]$ is a measurable function of $\left.N\right|_{\tau}$, respectively $\left.Z\right|_{\tau}$, so

$$
d_{\mathrm{TV}}(\mathcal{L}(N(\tau)), \mathcal{L}(Z(\tau))) \leq d_{\mathrm{TV}}\left(\mathcal{L}\left(\left.N\right|_{\tau}\right), \mathcal{L}\left(\left.Z\right|_{\tau}\right)\right)
$$

But as already observed, $N(\tau)$ is a sum of independent indicators and $Z(\tau)$ is a Poisson distribution having mean $M(\tau)$ common with $N(\tau)$. Thus, the lower bound of [4], Theorem 2, applies which is exactly the lower bound of the theorem.

We use Theorem 2.2 to prove convergence results through the following lemma which is easily proved from [18], Theorem 4.2.

Lemma 4.1. If $N^{(n)}$ is a sequence of processes on $\mathbb{R}_{+}^{\circ}$ such that there is a set $\left\{t_{i}: i \in \mathbb{N}\right\}$ with $0<t_{i} \uparrow \infty$ and for each $t_{i},\left.\left.N^{(n)}\right|_{t_{i}} \stackrel{d}{\longrightarrow} Z\right|_{t_{i}}$ as $n \rightarrow \infty$ in the vague topology for some process $Z$ on $\mathbb{R}_{+}^{\circ}$, then $N^{(n)} \stackrel{d}{\longrightarrow} Z$ as $n \rightarrow \infty$.

Proof of Corollary 2.5 (cf., $[11,17,28,29])$. As the mean measure of $N^{(n)}$ is different from that of $Z$, the natural proof we follow is to approximate $N^{(n)}$ with a Poisson process $Z^{(n)}$ having the same mean measure $\mathbb{E} Z^{(n)}(\cdot)=\mathbb{E} N^{(n)}(\cdot)$ and then approximate $Z^{(n)}$ by $Z$ using the condition $\lim _{n \rightarrow \infty} \mathbb{E} N^{(n)}(t)=L(t)$ for $t \in C(L)$. However, this last result does not seem to be noted anywhere, so we construct a proof using the natural metric for the vague topology defined in [3]. To this end, for each $t>0$, let $\chi_{t}$ (resp. $\mathcal{H}_{t} \subset \chi_{t}$ ) be the set of all finite Radon measures (resp. point measures) on $(0, t]$ and $\mathcal{K}_{t}$ be the set of all Lipschitz functions on $(0, t]$ with respect to the 
metric $d_{0}(x, y)=\min \{1,|x-y|\}$, that is, $\mathcal{K}_{t}=\left\{k:|k(x)-k(y)| \leq d_{0}(x, y), x, y \in(0, t]\right\}$. [3] introduce a Wasserstein metric $d_{1 t}$ for finite measures $\eta_{1}, \eta_{2} \in \chi_{t}$ as

$$
d_{1 t}\left(\eta_{1}, \eta_{2}\right)= \begin{cases}1, & \text { if } \eta_{1}(t) \neq \eta_{2}(t), \\ 0, & \text { if } \eta_{1}(t)=\eta_{2}(t)=0, \\ \frac{\sup _{k \in \mathcal{K}_{t}}\left|\int_{(0, t]} k(x) \eta_{1}(d x)-\int_{(0, t]} k(x) \eta_{2}(d x)\right|}{\eta_{1}(t)}, & \text { if } \eta_{1}(t)=\eta_{2}(t)>0 .\end{cases}
$$

Moreover, if $\eta_{1}=\sum_{j=1}^{m} \delta_{x_{j}}$ and $\eta_{2}=\sum_{j=1}^{m} \delta_{y_{j}}$ with $m>0$ and $\left\{x_{j}, y_{j}: 1 \leq j \leq m\right\} \subset(0, t]$, one can write

$$
d_{1 t}\left(\eta_{1}, \eta_{2}\right)=\min _{\pi}\left\{m^{-1} \sum_{j=1}^{m} d_{0}\left(x_{j}, y_{\pi(j)}\right)\right\},
$$

where $\pi$ ranges over all permutations of $\{1, \ldots, m\}$ [25], Section 2.2. The metric $d_{1 t}$ quantifies the vague (and the weak) topology on $\mathcal{H}_{t}$ [30]. [3] then introduce a Wasserstein metric $d_{2 t}$ induced by $d_{1 t}$ for two distributions $Q_{1}$ and $Q_{2}$ on $\mathcal{H}_{t}$ as

$$
d_{2 t}\left(Q_{1}, Q_{2}\right)=\sup _{f}\left|\int_{\mathcal{H}_{t}} f d Q_{1}-\int_{\mathcal{H}_{t}} f d Q_{2}\right|,
$$

where the supremum is taken over all $d_{1 t}$-Lipschitz functions on $\mathcal{H}_{t}$. Let $Z^{(n)}$ be a Poisson process on $\mathbb{R}_{+}^{\circ}$ with mean measure $M^{(n)}(\cdot):=\mathbb{E} N^{(n)}(\cdot)$. Now applying the triangle inequality, using the fact that $d_{2 t}\left(Q_{1}, Q_{2}\right) \leq d_{\mathrm{TV}}\left(Q_{1}, Q_{2}\right)$ and then applying the results [12], Theorem 1.5, and Theorem 2.2, we obtain for $t \in C(L)$,

$$
\begin{aligned}
d_{2 t} & \left(\mathcal{L}\left(\left.N^{(n)}\right|_{t}\right), \mathcal{L}\left(\left.Z\right|_{t}\right)\right) \\
& \leq d_{2 t}\left(\mathcal{L}\left(\left.N^{(n)}\right|_{t}\right), \mathcal{L}\left(\left.Z^{(n)}\right|_{t}\right)\right)+d_{2 t}\left(\mathcal{L}\left(\left.Z^{(n)}\right|_{t}\right), \mathcal{L}\left(\left.Z\right|_{t}\right)\right) \\
& \leq d_{\mathrm{TV}}\left(\mathcal{L}\left(\left.N^{(n)}\right|_{t}\right), \mathcal{L}\left(\left.Z^{(n)}\right|_{t}\right)\right)+d_{1 t}\left(\left.M^{(n)}\right|_{t} / M^{(n)}(t),\left.L\right|_{t} / L(t)\right)+\left|M^{(n)}(t)-L(t)\right| \\
& \leq \sum_{i \in \mathcal{I}_{n}} \mathbb{P}\left(Y_{n i} \leq t\right)^{2}+d_{1 t}\left(\left.M^{(n)}\right|_{t} / M^{(n)}(t),\left.L\right|_{t} / L(t)\right)+\left|M^{(n)}(t)-L(t)\right| \rightarrow 0
\end{aligned}
$$

as $n \rightarrow \infty$. Thus $\left.\left.N^{(n)}\right|_{t} \stackrel{d}{\longrightarrow} Z\right|_{t}$ as $n \rightarrow \infty$ for all $t \in C(L)$ and the claim follows from Lemma 4.1.

Proof of Proposition 2.6. Both assertions follow from writing

$$
|\xi|(r)=\sum_{i \in \mathcal{I}^{\xi}} \mathbf{1}_{0<\left|x_{i}\right| \leq r}
$$

The representation implies

$$
\int_{0}^{\infty} \mathbb{P}\left(0<\frac{h(r)}{S} \leq t\right)|\xi|(d r)=\sum_{i \in \mathcal{I}^{\xi}} \mathbb{P}\left(0<\frac{g\left(x_{i}\right)}{S} \leq t\right)=M(t) .
$$


For the second assertion, the indicator representation and the fact that

$$
h(x) \leq y \quad \Longleftrightarrow \quad x \leq h^{-1}(y)
$$

implies

$$
\mathbb{E}\left[|\xi|\left(h^{-1}(S t)\right)\right]=\mathbb{E} \sum_{i \in \mathcal{I}^{\xi}} \mathbf{1}_{0<\left|x_{i}\right| \leq h^{-1}(S t)}=\mathbb{E} \sum_{i \in \mathcal{I}^{\xi}} \mathbf{1}_{0<g\left(x_{i}\right) \leq S t}=M(t) .
$$

Proof of Theorem 2.7. That $Z$ is a Poisson process follows since $\left\{\left(x_{i}, S_{i}\right): x_{i} \in \Theta\right\}$ is a Poisson point process on $\mathbb{R}^{d} \times \mathbb{R}_{+}^{\circ}$ [20], page 55 , and the points of $Z$ are a measurable function of this process. The computation of the mean measure is straightforward and the bound on the total variation distance is easy to see from the coupling definition of the total variation distance since one can construct a coupling in such a way that if $\Theta=\Xi$ then $Z=N$.

Proof of Theorem 2.8. We use the following inequality relating the total variation distance of conditioned random variables to the unconditional; see, for example, [27], Section 3,

$$
d_{\mathrm{TV}}(\mathcal{L}(X), \mathcal{L}(Y)) \leq \mathbb{E} d_{\mathrm{TV}}(\mathcal{L}(X \mid W), \mathcal{L}(Y \mid W)) .
$$

We use this inequality to find

$$
d_{\mathrm{TV}}\left(\mathcal{L}\left(\left.Z\right|_{\tau}\right), \mathcal{L}\left(\left.N\right|_{\tau}\right)\right) \leq \mathbb{E} d_{\mathrm{TV}}\left(\mathcal{L}\left(\left.Z^{\Xi_{\mid}}\right|_{\tau}\right), \mathcal{L}\left(\left.N^{\Xi}\right|_{\tau}\right)\right)
$$

where $N^{\Xi}$ and $Z^{\Xi}$ denote the processes $N$ and $Z$ conditional on $\Xi$. Since $Z^{\Xi}$ is a Poisson process with mean measure $M^{\Xi}$, we apply Theorem 2.2 to obtain

$$
d_{\mathrm{TV}}\left(\mathcal{L}\left(\left.Z^{\Xi}\right|_{\tau}\right), \mathcal{L}\left(\left.N^{\Xi}\right|_{\tau}\right)\right) \leq \int_{\mathbb{R}^{d}} p_{i}^{(x)}(\tau)^{2} \Xi(d x)
$$

and now taking expectations and using (4.3) implies the theorem.

Proof of Theorem 1.1. The theorem follows easily from Corollary 2.5 and (2.2) once we establish that, for $t \in C(L)$,

$$
\lim _{\sigma \rightarrow \infty} \mathbb{E} D\left(h^{-1}(S(\sigma) t)\right)=\lim _{\sigma \rightarrow \infty} \mathbb{E}|\xi|\left(h^{-1}(S(\sigma) t)\right) .
$$

To show (4.4), let $\varepsilon>0$ and $r_{\varepsilon}$ be such that for $r \geq r_{\varepsilon}$

$$
1-\varepsilon<\frac{|\xi|(r)}{D(r)}<1+\varepsilon
$$

Then denoting the distribution function of $S(\sigma)$ by $F_{\sigma}$, we have

$$
\begin{aligned}
& \limsup _{\sigma \rightarrow \infty} \mathbb{E}|\xi|\left(h^{-1}(S(\sigma) t)\right) \\
& \quad \leq \limsup _{\sigma \rightarrow \infty} \int_{0}^{h\left(r_{\varepsilon}\right) / t}|\xi|\left(h^{-1}(s t)\right) F_{\sigma}(d s)+\limsup _{\sigma \rightarrow \infty} \int_{h\left(r_{\varepsilon}\right) / t}^{\infty}|\xi|\left(h^{-1}(s t)\right) F_{\sigma}(d s)
\end{aligned}
$$




$$
=\limsup _{\sigma \rightarrow \infty} \int_{h\left(r_{\varepsilon}\right) / t}^{\infty}|\xi|\left(h^{-1}(s t)\right) F_{\sigma}(d s)
$$

the equality is because $S(\sigma) \stackrel{\mathbb{P}}{\longrightarrow} 0$ and $\lim _{y \rightarrow 0} h^{-1}(y)=0$. Now using the definition (4.5) of $r_{\varepsilon}$ and noting that $s \geq h\left(r_{\varepsilon}\right) / t$ implies $h^{-1}(s t) \geq r_{\varepsilon}$, we bound (4.6) from above to find

$$
\begin{aligned}
\limsup _{\sigma \rightarrow \infty} \mathbb{E}|\xi|\left(h^{-1}(S(\sigma) t)\right) & \leq(1+\varepsilon) \limsup _{\sigma \rightarrow \infty} \int_{h\left(r_{\varepsilon}\right) / t}^{\infty} D\left(h^{-1}(s t)\right) F_{\sigma}(d s) \\
& \leq(1+\varepsilon) \limsup _{\sigma \rightarrow \infty} \int_{0}^{\infty} D\left(h^{-1}(s t)\right) F_{\sigma}(d s) \\
& =(1+\varepsilon) \lim _{\sigma \rightarrow \infty} \mathbb{E} D\left(h^{-1}(S(\sigma) t)\right) .
\end{aligned}
$$

As $\varepsilon$ is arbitrary, this yields

$$
\limsup _{\sigma \rightarrow \infty} \mathbb{E}|\xi|\left(h^{-1}(S(\sigma) t)\right) \leq \lim _{\sigma \rightarrow \infty} \mathbb{E} D\left(h^{-1}(S(\sigma) t)\right) .
$$

Similarly,

$$
\liminf _{\sigma \rightarrow \infty} \mathbb{E}|\xi|\left(h^{-1}(S(\sigma) t)\right) \geq(1-\varepsilon) \liminf _{\sigma \rightarrow \infty} \int_{h\left(r_{\varepsilon}\right) / t}^{\infty} D\left(h^{-1}(s t)\right) F_{\sigma}(d s) .
$$

But again using that $S(\sigma) \rightarrow 0$ in probability, $\lim _{y \rightarrow 0} h^{-1}(y)=0$, and now also that $\lim _{r \rightarrow 0} D(r) \rightarrow 0$,

$$
\lim _{\sigma \rightarrow \infty} \int_{h\left(r_{\varepsilon}\right) / t}^{\infty} D\left(h^{-1}(s t)\right) F_{\sigma}(d s)=\lim _{\sigma \rightarrow \infty} \int_{0}^{\infty} D\left(h^{-1}(s t)\right) F_{\sigma}(d s),
$$

and combining this with (4.8) implies

$$
\liminf _{\sigma \rightarrow \infty} \mathbb{E}|\xi|\left(h^{-1}(S(\sigma) t)\right) \geq(1-\varepsilon) \lim _{\sigma \rightarrow \infty} \mathbb{E} D\left(h^{-1}(S(\sigma) t)\right)
$$

Since $\varepsilon$ was arbitrary, we have that

$$
\liminf _{\sigma \rightarrow \infty} \mathbb{E}|\xi|\left(h^{-1}(S(\sigma) t)\right) \geq \lim _{\sigma \rightarrow \infty} \mathbb{E} D\left(h^{-1}(S(\sigma) t)\right)
$$

which, together with (4.7), proves (4.4).

Proof of Theorem 2.10. For the sake of brevity, write $p_{\sigma}^{(x)}(t)=\mathbb{P}(0<g(x) / S(\sigma) \leq t)$, $M_{\sigma}^{\Xi}(t)=\int_{\mathbb{R}^{d}} p_{\sigma}^{(x)}(t) \Xi(d x)$ and $M_{\sigma}^{\Lambda}(t)=\int_{\mathbb{R}^{d}} p_{\sigma}^{(x)}(t) \Lambda(d x)$. We divide the proof into three steps.

(i) $M_{\sigma}^{\Xi} \stackrel{d}{\longrightarrow} L$ in the vague topology as $\sigma \rightarrow \infty$. 
To show the claim, from [18], Theorem 4.2, it suffices to show that for each continuous function $f: \mathbb{R}_{+}^{\circ} \rightarrow \mathbb{R}_{+}$with compact support and bounded continuous first derivative,

$$
\int_{\mathbb{R}_{+}^{\circ}} f(t) d M_{\sigma}^{\Xi}(t) \stackrel{d}{\longrightarrow} \int_{\mathbb{R}_{+}^{\circ}} f(t) d L(t) .
$$

Now, let $F_{\sigma}$ be the distribution function of $S(\sigma)$ and let $0<a<b<\infty$ such that $b \in C(L)$ and the support of $f$ is contained in $[a, b]$ such that $f(b)=f(a)=0$. It follows from (2.2) that

$$
M_{\sigma}^{\Xi}(t)=\int_{\mathbb{R}_{+}^{\circ}}|\Xi|\left(h^{-1}(s t)\right) d F_{\sigma}(s)
$$

and, by taking expectation,

$$
M_{\sigma}^{\Lambda}(t)=\int_{\mathbb{R}_{+}^{\circ}}|\Lambda|\left(h^{-1}(s t)\right) d F_{\sigma}(s) .
$$

Using Fubini's theorem and noting $f(b)=f(a)=0$, we have

$$
\int_{\mathbb{R}_{+}^{\circ}} f(t) d M_{\sigma}^{\Xi}(t)=\int_{\mathbb{R}_{+}^{\circ}} \int_{a}^{t} f^{\prime}(s) d s d M_{\sigma}^{\Xi}(t)=-\int_{a}^{b} M_{\sigma}^{\Xi}(t) f^{\prime}(t) d t
$$

and hence

$$
\begin{aligned}
\int_{\mathbb{R}_{+}^{\circ}} f(t) d M_{\sigma}^{\Xi}(t)= & -\int_{0}^{\infty} \int_{a}^{b}|\Xi|\left(h^{-1}(s t)\right) f^{\prime}(t) d t d F_{\sigma}(s) \\
= & -\int_{\theta_{0}}^{\infty} \int_{a}^{b}\left[|\Xi|\left(h^{-1}(s t)\right)-|\Lambda|\left(h^{-1}(s t)\right)\right] f^{\prime}(t) d t d F_{\sigma}(s) \\
& -\int_{0}^{\infty} \int_{a}^{b}|\Lambda|\left(h^{-1}(s t)\right) f^{\prime}(t) d t d F_{\sigma}(s) \\
& +\int_{0}^{\theta_{0}} \int_{a}^{b}|\Lambda|\left(h^{-1}(s t)\right) f^{\prime}(t) d t d F_{\sigma}(s) \\
& -\int_{0}^{\theta_{0}} \int_{a}^{b}|\Xi|\left(h^{-1}(s t)\right) f^{\prime}(t) d t d F_{\sigma}(s) \\
= & \text { (I) }+(\mathrm{II})+(\mathrm{III})+(\mathrm{IV}),
\end{aligned}
$$

where $\theta_{0}$ is chosen such that

$$
\frac{\operatorname{Var}\left(|\Xi|\left(h^{-1}(s a)\right)\right)}{|\Lambda|\left(h^{-1}(s a)\right)^{2}} \leq 1, \quad \forall s \geq \theta_{0} .
$$

We complete the proof of (4.9) by showing that, as $\sigma \rightarrow \infty$, (a) (I) $\stackrel{\mathbb{P}}{\longrightarrow} 0$; (b) (II) $\rightarrow$ $\int_{\mathbb{R}_{+}^{\circ}} f(t) d L(t) ;(\mathrm{c})(\mathrm{III}) \rightarrow 0$ and $(\mathrm{d})(\mathrm{IV}) \stackrel{\mathbb{P}}{\longrightarrow} 0$. 
(a) This is the part of the proof that crucially relies on $\operatorname{Var}(|\Xi|(r)) /(|\Lambda|(r))^{2} \rightarrow 0$; see just below (4.11). Let $v_{\sigma}$ be the variance of (I), write $\Xi^{\prime}(t):=|\Xi|\left(h^{-1}(t)\right)$ and $\Lambda^{\prime}(t):=|\Lambda|\left(h^{-1}(t)\right)$, then using the geometric-arithmetic mean inequality $A B \leq\left(A^{2}+B^{2}\right) / 2$ in the first inequality and the symmetry in the third equality, we have

$$
\begin{aligned}
v_{\sigma}= & \mathbb{E}\left\{\int_{\theta_{0}}^{\infty} \int_{a}^{b}\left[\Xi^{\prime}(s t)-\Lambda^{\prime}(s t)\right] f^{\prime}(t) d t d F_{\sigma}(s)\right\}^{2} \\
= & \mathbb{E} \int_{\theta_{0}}^{\infty} \int_{a}^{b} \int_{\theta_{0}}^{\infty} \int_{a}^{b}\left(\frac{\Xi^{\prime}\left(s_{1} t_{1}\right)}{\Lambda^{\prime}\left(s_{1} t_{1}\right)}-1\right) f^{\prime}\left(t_{1}\right)\left(\frac{\Xi^{\prime}\left(s_{2} t_{2}\right)}{\Lambda^{\prime}\left(s_{2} t_{2}\right)}-1\right) f^{\prime}\left(t_{2}\right) \\
& \times \Lambda^{\prime}\left(s_{1} t_{1}\right) d t_{1} d F_{\sigma}\left(s_{1}\right) \Lambda^{\prime}\left(s_{2} t_{2}\right) d t_{2} d F_{\sigma}\left(s_{2}\right) \\
\leq & \mathbb{E} \int_{\theta_{0}}^{\infty} \int_{a}^{b} \int_{\theta_{0}}^{\infty} \int_{a}^{b} \frac{1}{2}\left\{\left[\left(\frac{\Xi^{\prime}\left(s_{1} t_{1}\right)}{\Lambda^{\prime}\left(s_{1} t_{1}\right)}-1\right) f^{\prime}\left(t_{1}\right)\right]^{2}\right. \\
& \left.+\left[\left(\frac{\Xi^{\prime}\left(s_{2} t_{2}\right)}{\Lambda^{\prime}\left(s_{2} t_{2}\right)}-1\right) f^{\prime}\left(t_{2}\right)\right]^{2}\right\} \\
& \times \Lambda^{\prime}\left(s_{1} t_{1}\right) d t_{1} d F_{\sigma}\left(s_{1}\right) \Lambda^{\prime}\left(s_{2} t_{2}\right) d t_{2} d F_{\sigma}\left(s_{2}\right) \\
= & \int_{\theta_{0}}^{\infty} \int_{a}^{b} \int_{\theta_{0}}^{\infty} \int_{a}^{b} \frac{\operatorname{Var}\left(\Xi^{\prime}\left(s_{1} t_{1}\right)\right)}{\Lambda^{\prime}\left(s_{1} t_{1}\right)^{2}} f^{\prime}\left(t_{1}\right)^{2} \Lambda^{\prime}\left(s_{1} t_{1}\right) d t_{1} d F_{\sigma}\left(s_{1}\right) \Lambda^{\prime}\left(s_{2} t_{2}\right) d t_{2} d F_{\sigma}\left(s_{2}\right) \\
\leq & \left\|f^{\prime}\right\|^{2}(b-a) \mathbb{E} \Lambda^{\prime}(S(\sigma) b) \int_{\theta_{0}}^{\infty} \Lambda^{\prime}\left(s_{1} b\right) \int_{a}^{b} \frac{\operatorname{Var}\left(\Xi^{\prime}\left(s_{1} t_{1}\right)\right)}{\Lambda^{\prime}\left(s_{1} t_{1}\right)^{2}} d t_{1} d F_{\sigma}\left(s_{1}\right),
\end{aligned}
$$

where $\left\|f^{\prime}\right\|=\sup _{t \in \mathbb{R}_{+}^{\circ}}\left|f^{\prime}(t)\right|$. For each $\epsilon>0$, let $T_{\epsilon}>\theta_{0}$ such that

$$
\frac{\operatorname{Var}\left(\Xi^{\prime}(s a)\right)}{\Lambda^{\prime}(s a)^{2}} \leq \epsilon, \quad \forall s \geq T_{\epsilon} .
$$

It follows from (4.11) that

$$
\begin{aligned}
v_{\sigma} & \leq\left\|f^{\prime}\right\|^{2}(b-a) \mathbb{E} \Lambda^{\prime}(S(\sigma) b)\left(\int_{\theta_{0}}^{T_{\epsilon}} \int_{a}^{b}+\epsilon \int_{T_{\epsilon}}^{\infty} \int_{a}^{b}\right) \Lambda^{\prime}\left(s_{1} b\right) d t_{1} d F_{\sigma}\left(s_{1}\right) \\
& \leq\left\|f^{\prime}\right\|^{2}(b-a)^{2} \mathbb{E} \Lambda^{\prime}(S(\sigma) b)\left(\mathbb{E} \Lambda^{\prime}(S(\sigma) b) \mathbf{1}_{S(\sigma) \leq T_{\epsilon}}+\epsilon \mathbb{E} \Lambda^{\prime}(S(\sigma) b)\right) .
\end{aligned}
$$

Using that $S(\sigma) \stackrel{\mathbb{P}}{\longrightarrow} 0, \quad \Lambda^{\prime}(S(\sigma) b) \mathbf{1}_{S(\sigma) \leq T_{\epsilon}} \leq \Lambda^{\prime}\left(T_{\epsilon} b\right)<\infty, \quad \lim _{t \downarrow} h^{-1}(t)=0$ and $\lim _{r \downarrow 0}|\Lambda|(r)=0$, we apply the bounded convergence theorem to obtain

$$
\lim _{\sigma \rightarrow \infty} \mathbb{E} \Lambda^{\prime}(S(\sigma) b) \mathbf{1}_{S(\sigma) \leq T_{\epsilon}}=0
$$

which, together with (4.12), ensures

$$
\limsup _{\sigma \rightarrow \infty} v_{\sigma} \leq\left\|f^{\prime}\right\|^{2}(b-a)^{2} \epsilon L(b)^{2} .
$$


This yields $\lim _{\sigma \rightarrow \infty} v_{\sigma}=0$ due to the arbitrariness of $\epsilon$.

(b) Applying the dominated convergence theorem, we have

$$
\begin{aligned}
& -\int_{0}^{\infty} \int_{a}^{b}|\Lambda|\left(h^{-1}(s t)\right) f^{\prime}(t) d t d F_{\sigma}(s) \\
& =-\int_{a}^{b} \mathbb{E}|\Lambda|\left(h^{-1}(S(\sigma) t)\right) f^{\prime}(t) d t \\
& \rightarrow-\int_{a}^{b} f^{\prime}(t) L(t) d t=\int_{a}^{b} f(t) d L(t)=\int_{\mathbb{R}_{+}^{\circ}} f(t) d L(t),
\end{aligned}
$$

where the penultimate equality is due to Fubini's theorem similar to (4.10).

(c) Use the same reasoning as that for (4.13); as $\sigma \rightarrow \infty$,

$$
\int_{0}^{\theta_{0}} \int_{a}^{b}|\Lambda|\left(h^{-1}(s t)\right)\left|f^{\prime}(t)\right| d t d F_{\sigma}(s) \leq\left\|f^{\prime}\right\|(b-a) \mathbb{E}|\Lambda|\left(h^{-1}(S(\sigma) b)\right) \mathbf{1}_{S(\sigma) \leq \theta_{0}} \rightarrow 0 .
$$

(d) It follows from (c) that, as $\sigma \rightarrow \infty$,

$\mathbb{E} \int_{0}^{\theta_{0}} \int_{a}^{b}|\Xi|\left(h^{-1}(s t)\right)\left|f^{\prime}(t)\right| d t d F_{\sigma}(s) \leq\left\|f^{\prime}\right\|(b-a) \mathbb{E}|\Lambda|\left(h^{-1}(S(\sigma) b)\right) \mathbf{1}_{S(\sigma) \leq \theta_{0}} \rightarrow 0$.

At this point the proof follows along the lines of Corollary 2.5.

(ii) With the notation in the proof of Corollary 2.5, let $Z^{L}$ be a Poisson process with mean measure $L$, then for each $t>0$,

$$
\begin{aligned}
& d_{2 t}\left(\mathcal{L}\left(\left.N^{(\sigma)}\right|_{t}\right), \mathcal{L}\left(\left.Z^{L}\right|_{t}\right)\right) \\
& \quad \leq \int_{\mathbb{R}^{d}} p_{\sigma}^{(x)}(t)^{2} \Lambda(d x)+\mathbb{E} d_{1 t}\left(\left.M_{\sigma}^{\Xi}\right|_{t} / M_{\sigma}^{\Xi}(t),\left.L\right|_{t} / L(t)\right)+\mathbb{E} 1 \wedge\left|M_{\sigma}^{\Xi}(t)-L(t)\right| .
\end{aligned}
$$

To show (ii), let $Z_{\sigma}^{\Xi}$ be a Cox process directed by the mean measure $M_{\sigma}^{\Xi}$, then

$$
d_{2 t}\left(\mathcal{L}\left(\left.N^{(\sigma)}\right|_{t}\right), \mathcal{L}\left(\left.Z_{\sigma}^{\Xi}\right|_{t}\right)\right) \leq d_{\mathrm{TV}}\left(\mathcal{L}\left(\left.N^{(\sigma)}\right|_{t}\right), \mathcal{L}\left(\left.Z_{\sigma}^{\Xi}\right|_{t}\right)\right)
$$

and the first term in the upper bound of (4.14) comes from Theorem 2.8. For a Poisson process $\tilde{Z}$ on $\mathbb{R}_{+}^{\circ}$ with mean measure $\lambda$, [12], Theorem 1.5 , gives

$$
d_{2 t}\left(\mathcal{L}\left(\left.\tilde{Z}\right|_{t}\right), \mathcal{L}\left(\left.Z^{L}\right|_{t}\right)\right) \leq d_{1 t}\left(\left.\lambda\right|_{t} / \lambda(t),\left.L\right|_{t} / L(t)\right)+1 \wedge|\lambda(t)-L(t)|,
$$

where 1 is because $d_{2 t} \leq 1$. Hence,

$$
\begin{aligned}
& d_{2 t}\left(\mathcal{L}\left(\left.Z_{\sigma}^{\Xi}\right|_{t}\right), \mathcal{L}\left(\left.Z^{L}\right|_{t}\right)\right)=\sup _{f}\left|\mathbb{E} f\left(\left.Z_{\sigma}^{\Xi}\right|_{t}\right)-\mathbb{E} f\left(\left.Z^{L}\right|_{t}\right)\right| \\
& \leq \mathbb{E} \sup _{f}\left|\mathbb{E}\left[f\left(\left.Z_{\sigma}^{\Xi}\right|_{t}\right) \mid \Xi\right]-\mathbb{E} f\left(\left.Z^{L}\right|_{t}\right)\right| \\
& \leq \mathbb{E} d_{1 t}\left(\left.M_{\sigma}^{\Xi}\right|_{t} / M_{\sigma}^{\Xi}(t),\left.L\right|_{t} / L(t)\right)+\mathbb{E} 1 \wedge\left|M_{\sigma}^{\Xi}(t)-L(t)\right|,
\end{aligned}
$$


where $f$ ranges over all $d_{1 t}$-Lipschitz functions on $\mathcal{H}_{t}$. This gives the remaining two terms in the upper bound of (4.14).

(iii) $N^{(\sigma)}$ converges weakly to a Poisson process with mean measure $L$.

To prove this claim, Lemma 4.1 implies it suffices to show that for each fixed $t \in C(L)$, the upper bound of (4.14) converges to 0 as $\sigma \rightarrow \infty$. For the first term, we have, for each $r>0$,

$$
\int_{\mathbb{R}^{d}} p_{\sigma}^{(x)}(t)^{2} \Lambda(d x) \leq|\Lambda|(r)+\mathbb{P}(S(\sigma) \geq h(r) / t) \mathbb{E}|\Lambda|\left(h^{-1}(S(\sigma) t)\right),
$$

which, together with the assumption $S(\sigma) \stackrel{\mathbb{P}}{\longrightarrow} 0$, implies

$$
\limsup _{\sigma \rightarrow \infty} \int_{\mathbb{R}^{d}} p_{\sigma}^{(x)}(t)^{2} \Lambda(d x) \leq|\Lambda|(r)
$$

Since $r$ is arbitrary and $\lim _{r \rightarrow 0}|\Lambda|(r)=0$, we obtain $\lim _{\sigma \rightarrow \infty} \int_{\mathbb{R}^{d}} p_{\sigma}^{(x)}(t)^{2} \Lambda(d x)=0$. For the second term in the upper bound of (4.14), as $d_{1 t}\left(\cdot,\left.L\right|_{t} / L(t)\right)$ is a continuous function on

$$
\left\{v: v \text { is right continuous and nondecreasing on }(0, t] \text { with } \lim _{s \downarrow 0} v(s)=0, v(t)=1\right\}
$$

that equals zero at $\left.L\right|_{t} / L(t)$, it follows from (i) and the continuity theorem that, as $\sigma \rightarrow \infty$,

$$
d_{1 t}\left(\left.M_{\sigma}^{\Xi}\right|_{t} / M_{\sigma}^{\Xi}(t),\left.L\right|_{t} / L(t)\right) \stackrel{d}{\longrightarrow} 0
$$

and hence $\mathbb{E} d_{1 t}\left(\left.M_{\sigma}^{\Xi}\right|_{t} / M_{\sigma}^{\Xi}(t),\left.L\right|_{t} / L(t)\right) \rightarrow 0$. The third term converges to 0 due to (i) and the bounded convergence theorem.

\section{Final remarks}

We show that under general conditions the wireless network signals appear to different observers at different times as different Poisson processes or different realizations of the same Cox process. This line of work strongly suggests that given the network is sufficiently large and stationary (or just isotropic) with strong enough random propagation effects such as fading and shadowing, then the signal strengths can be modeled directly as a Poisson or Cox process on the real line and the details of the distribution of the positioning of transmitters on the plane can be safely ignored. From the results presented here, there are many further directions of study: For a given transmitter configuration, do some fading models induce a propagation process significantly closer to Poisson than others? How do our results translate to functions of the propagation process, for example, the signal-to-interference ratio discussed in the introduction (cf., [19])? Can our results be extended to models with short range (spatial) dependence between the fading variables? 


\section{Acknowledgements}

This work was supported by Australian Research Council grants DP110101663 (H.P. Keeler), DP120102398 (A. Xia), and DP150101459 (N. Ross and A. Xia). We thank the referee and Associate Editor for helpful comments.

\section{References}

[1] Baccelli, F. and Błaszczyszyn, B. (2009). Stochastic Geometry and Wireless Networks - Volume 1: Theory. Now Publishers Inc.

[2] Baccelli, F. and Błaszczyszyn, B. (2009). Stochastic Geometry and Wireless Networks - Volume 2: Application. Now Publishers Inc.

[3] Barbour, A.D. and Brown, T.C. (1992). Stein's method and point process approximation. Stochastic Process. Appl. 43 9-31. MR1190904

[4] Barbour, A.D. and Hall, P. (1984). On the rate of Poisson convergence. Math. Proc. Cambridge Philos. Soc. 95 473-480. MR0755837

[5] Barbour, A.D., Holst, L. and Janson, S. (1992). Poisson Approximation. Oxford Studies in Probability 2. Oxford Univ. Press: London. MR1163825

[6] Błaszczyszyn, B., Karray, M.K. and Keeler, H.P. (2013). Using Poisson processes to model lattice cellular networks. In INFOCOM, 2013 Proceedings IEEE 773-781. IEEE.

[7] Błaszczyszyn, B., Karray, M.K. and Keeler, H.P. (2015). Wireless networks appear Poissonian due to strong shadowing. IEEE Trans. Wirel. Commun. 14 4379-4390.

[8] Błaszczyszyn, B., Karray, M.K. and Klepper, F.X. (2010). Impact of the geometry, path-loss exponent and random shadowing on the mean interference factor in wireless cellular networks. In Third Joint IFIP Wireless and Mobile Networking Conference (WMNC).

[9] Błaszczyszyn, B. and Keeler, H.P. (2015). Studying the SINR process of the typical user in Poisson networks using its factorial moment measures. IEEE Trans. Inform. Theory 61 6774-6794. MR3430722

[10] Błaszczyszyn, B. and Yogeshwaran, D. (2015). Clustering comparison of point processes, with applications to random geometric models. In Stochastic Geometry, Spatial Statistics and Random Fields. Lecture Notes in Math. 2120 31-71. Cham: Springer. MR3330572

[11] Brown, T.C. (1979). Position dependent and stochastic thinning of point processes. Stochastic Process. Appl. 9 189-193.

[12] Brown, T.C. and Xia, A. (1995). On metrics in point process approximation. Stoch. Stoch. Rep. 52 247-263. MR1381671

[13] Franceschetti, M., Bruck, J. and Schulman, L.J. (2004). A random walk model of wave propagation. IEEE Trans. Antennas and Propagation 52 1304-1317.

[14] Haenggi, M. (2008). A geometric interpretation of fading in wireless networks: Theory and applications. IEEE Trans. Inform. Theory $\mathbf{5 4}$ 5500-5510. MR2590524

[15] Haenggi, M. (2012). Stochastic Geometry for Wireless Networks. Cambridge: Cambridge Univ. Press.

[16] Haenggi, M., Andrews, J.G., Baccelli, F., Dousse, O. and Franceschetti, M. (2009). Stochastic geometry and random graphs for the analysis and design of wireless networks. IEEE J. Sel. Areas Commun. 27 1029-1046.

[17] Kallenberg, O. (1975). Limits of compound and thinned point processes. J. Appl. Probab. 12 269-278. MR0391251

[18] Kallenberg, O. (1983). Random Measures, 3rd ed. London: Academic Press. MR0818219 
[19] Keeler, H.P. and Blaszczyszyn, B. (2014). SINR in wireless networks and the two-parameter PoissonDirichlet process. IEEE Wireless Communications Letters 3 525-528.

[20] Kingman, J.F.C. (1993). Poisson Processes. Oxford Studies in Probability 3. London: Oxford Univ. Press.

[21] Lee, C.-H., Shih, C.-Y. and Chen, Y.-S. (2013). Stochastic geometry based models for modeling cellular networks in urban areas. Wirel. Netw. 19 1063-1072.

[22] Le Cam, L. (1960). An approximation theorem for the Poisson binomial distribution. Pacific J. Math. 10 1181-1197.

[23] Miyoshi, N. and Shirai, T. (2014). A cellular network model with Ginibre configured base stations. Adv. in Appl. Probab. 46 832-845. MR3254344

[24] Miyoshi, N. and Shirai, T. (2014). Cellular networks with $\alpha$-Ginibre configurated base stations. In The Impact of Applications on Mathematics. Math. Ind. (Tokyo) 1211-226. Tokyo: Springer. MR3445552

[25] Rachev, S.T. (1984). The Monge-Kantorovich mass transference problem and its stochastic applications. Theory Probab. Appl. 29 647-676.

[26] Reig, J. and Rubio, L. (2013). Estimation of the composite fast fading and shadowing distribution using the log-moments in wireless communications. IEEE Trans. Wirel. Commun. 12 3672-3681.

[27] Röllin, A. and Ross, N. (2015). Local limit theorems via Landau-Kolmogorov inequalities. Bernoulli 21 851-880.

[28] Schuhmacher, D. (2005). Distance estimates for Poisson process approximations of dependent thinnings. Electron. J. Probab. 10 165-201.

[29] Schuhmacher, D. (2009). Distance estimates for dependent thinnings of point processes with densities. Electron. J. Probab. 14 1080-1116.

[30] Xia, A. (2005). Stein's method and Poisson process approximation. In An Introduction to Stein's Method (A.D. Barbour and L.H.Y. Chen, eds.) 115-181. Singapore: World Scientific Press.

[31] Zhang, X. and Andrews, J.G. (2015). Downlink cellular network analysis with multi-slope path loss models. IEEE Transactions on Communications 63 1881-1894.

Received September 2015 and revised August 2016 